\title{
Editorial
}

\section{Sobrediagnóstico e tratamento excessivo: Médicos generalistas - é hora de uma revolução na medicina}

Julian Treadwell. Clínico Geral, Hindon Surgery, Willshire; Vice-Presidente, Grupo Permanente sobre Sobrediagnóstico de RCGP; Membro do Conselho Editorial, Boletim de Drogas e Medicamentos; Avaliador de Clínicos Gerais, NHSE South Central. jools.jt@zen.co.uk (Autor correspondente) Margaret McCartney. Clínica geral, Fulton Street Medical Centre, Glasgow; Presidente, Grupo Permanente sobre Sobrediagnóstico de RCGP.

\section{“A RBMFC publica um artigo mensalmente traduzido do BJGP”}

"A multimorbilidade iatrogênica cria um fardo de polimedicação prejudicial para pacientes e seus cuidadores"

“...esperamos que, ao redistribuirmos nosso tempo de intervenções de pouco valor e em direção a intervenções de valor alto, nós desfrutaremos de trabalhos muito mais gratificantes, compartilhando decisões com nossos pacientes e reivindicando o nosso papel como especialistas gerais."

Práticas médicas ineficientes e prejudiciais sempre estiveram conosco, mas a escala e institucionalização do sobrediagnóstico e do tratamento excessivo se expandiram exponencialmente nas últimas décadas.

Este tema tem sido articulado em movimentos mundiais, tais como as conferências para Prevenção de Sobrediagnóstico, e campanhas como "Medicina em Demasia" ("Too Much Medicine"), do BMJ, "Menos é Mais" ("Less is More") da JAMA, o movimento italiano "Desacelerem a Medicina" ("Slow Medicine") e o projeto "Escolhendo com Inteligência” (“Choosing Wisely”), dos EUA (e agora internacional).

Como citar: Treadwell J, McCartney M. Sobrediagnóstico e tratamento excessivo: Médicos generalistas - é hora de uma revolução na medicina. Rev Bras Med Fam Comunidade. 2016;11(38):1-5. http://dx.doi.org/10.5712/rbmfc11(38)1400
Documento original publicado no British Journal of General Practice, março de 2016. 
Em 2014, a Royal College of General Pratictioners (RGCP) estabeleceu seu Grupo Permanente de Sobrediagnóstico [Apoiando Decisões Compartilhadas em Cuidados Médicos].

O sobrediagnóstico foi definido como “...quando pessoas sem sintomas são diagnosticadas com uma doença que, no fim das contas, não as causará sintomas ou morte precoce", e também é usado como uma designação abrangente para incluir "...os problemas relacionados à medicalização excessiva e o tratamento excessivo subsequente, desvirtuamento de diagnóstico, alteração de limiares e mercantilização de doença". ${ }^{1}$ Alguns autores usam a Too Much Medicine para englobar estas questões mais amplas. ${ }^{2}$

Os mecanismos do sobrediagnóstico são bem descritos. A tecnologia avançada permite a detecção de doenças em seus estágios iniciais, ou estados "pré-doença". Boas intenções e interesses ocultos se combinam a limiares mais baixos para tratamento e intervenção de modo que segmentos cada vez maiores da população assintomática adquiram diagnósticos, fatores de risco ou rótulos de doenças. Este processo é apoiado por medo de judicialização médica e por pagamentos e indicadores de desempenho que recompensam atividades excessivas. Isto levou a uma cultura de protocolos que evoluiu involuntariamente para espremer a tomada de decisões customizada e focada em pessoas. Subjacente a tudo isto estão as narrativas, profundamente intuitivas, ao redor dos supostos benefícios da detecção precoce e intervenção que são difíceis de anular, igualmente para profissionais e público.

Isto leva a perigos reais. O fardo psicológico de adquirir um título de doença pode parecer difícil de quantificar - parcialmente por causa de pesquisas insuficientes - para algo como doença renal crônica (DRC). Entretanto, se torna mais óbvio quando se considera um diagnóstico falso-positivo de demência ou um sobrediagnóstico de rastreamento de câncer de mama. Os pacientes são expostos aos danos do tratamento, dos mais moderados aos fatais, e é inevitável que haja desperdício de recursos em grande escala. A questão crítica do custo de oportunidade pode ser levantada, mas é desconsiderada em decisões de custo-efetividade. ${ }^{4}$

Nada disto é simples; o sobrediagnóstico e o tratamento excessivo surgem como consequência de atividades que têm um grau de benefício para certas pessoas. Dilemas surgem sobre como danos e benefícios são pesados ou percebidos, e como oferecer escolhas reais para pacientes em vez de simplesmente apresentar aquelas opções que o contrato ou os protocolos direcionam.

Acreditamos que os generalistas (MFC) possuem expertise específica aqui. Guias da prática clínica tendem a vir de pesquisa especializada. Especialistas que lidam com condições individuais podem ter em mente desfechos específicos para doenças, mas essas precisam ser priorizadas pelo paciente no contexto de seus problemas de saúde mais amplos e a percepção de risco e benefício. Para o médico, estes desfechos precisam ser priorizados com o conhecimento das consequências de intervenções sobre a população geral ou o sistema. Os generalistas recebem a responsabilidade de fazer intervenções no nível da população para atingir objetivos específicos, mas tratamos indivíduos que podem, correta e razoavelmente, ter um ponto de vista diferente.

\section{Pseudosoluções para problemas difíceis}

Problemas de saúde pública sem soluções fáceis são terreno fértil para atividades excessivas em grande escala nos cuidados primários. Exames de check-up do NHS (sistema de saúde britânico) foram introduzidos nacionalmente com um incentivo financeiro como uma solução aparente para a doença 
metabólica, apesar de quatro décadas de evidência provarem que estes programas não afetam a morbilidade ou mortalidade populacional. ${ }^{5-7}$

A triagem para demência não foi recomendada pelo Comitê de Triagem Nacional do Reino Unido (UK National Screening Committee), pois possui risco significativo de danos com possíveis diagnósticos falso-positivos de demência, ${ }^{8}$ sem falar do custo de oportunidade criado pelo inevitável "sequestro da consulta".

Esta atividade pode parecer sensível, mas faltam evidências e distrai da necessidade de soluções mais desafiadoras como a abordagem do ambiente obesogênico ou a melhoria do cuidado social para pessoas com demência.

\section{Alteração de limiares ou desvirtuamento de indicações}

Limiares para rótulos como diabetes ou doença renal crônica são criados com base na identificação de riscos acima da média, e assim as populações-alvo ficam ainda mais perto de conter metade dos adultos mais velhos. Isto tente a preceder a evidência de efeitos sobre resultados de desfecho para os novos "pacientes" na extremidade moderada do espectro de risco. 9,10

Limiares de tratamentos inferiores sempre criam um aumento no número de pacientes tomando medicamentos sem benefício nenhum para que somente alguns possam tê-lo. Ao anunciar seu mais recente protocolo de dislipidemia, o Instituto Nacional para Excelência em Saúde e Cuidado ("National Institute for Health and Care Excellence", NICE) estimou que, caso todas as pessoas elegíveis recebessem tratamento, poderíamos prevenir 28.000 ataques cardíacos, 16.000 derrames e 8.000 mortes ao longo de três anos. Nós também daríamos estatinas para 4.448.000 pacientes com benefício nenhum. ${ }^{11}$

Buscar reduzir o sobrediagnóstico e o tratamento excessivo não implica em niilismo terapêutico, tampouco em um desejo de abandonar a medicina preventiva. Ao invés disto, nos força a buscar uma compreensão da base de evidências que auxilia pacientes a fazer escolhas de maneira útil e cognitiva acerca dos benefícios e danos.

Os generalistas (MFC) não se deparam apenas com as consequências da medicalização excessiva, mas também carregam uma carga de trabalho extra causada por isto. A multimorbilidade iatrogênica cria um fardo de polimedicação prejudicial para pacientes e seus cuidadores. Os médicos focados em uma condição podem não ter a visão geral, holística e generalista necessária para ajudar o paciente a diferenciar intervenções valiosas daquelas sem valor. A defesa de nossos pacientes contra os perigos de "Too Much Medicine" precisa acontecer não só no consultório, mas também com criação de políticas locais e nacionais.

ARCGP tem diversos mecanismos a seu dispor, incluindo o desenvolvimento de fontes de evidência e a melhoria de nossos relacionamentos com colegas especialistas e órgãos externos. Em 2015, o Conselho da RCGP aprovou um documento de orientação quanto ao sobrediagnóstico ${ }^{12}$ com uma recomendação de que cinco tópicos fossem divulgados pela Associação Britânica dos Médicos de Família (RCGP) de modo a reduzir o risco de sobremedicação:

- Apresentar evidências de maneira que permita a tomada compartilhada de decisões e o envolvimento dos pacientes (risco absoluto, número necessário para tratar);

- Clareza sobre quais populações as evidências razoavelmente se aplicam;

- Abertura sobre a incerteza das evidências; 
- Afirmar quais propostas para triagens foram aprovadas pelo Comitê Nacional de Triagem (National Screening Committee); e

- $\quad$ Finalmente, que as declarações de interesse sejam tornadas públicas.

Entretanto, o recurso mais valioso é o nosso tempo, e esperamos que, ao redistribuirmos nosso tempo de intervenções de pouco valor em direção a intervenções de alto valor, nós desfrutaremos de um trabalho muito mais gratificante, compartilhando decisões com nossos pacientes e reivindicando o nosso papel como especialistas gerais.

Para isto, devemos ser claros a respeito de custos de oportunidade. Todas as novas inovações ou intervenções que forem sugeridas para generalistas (MFC) deverão ser acompanhadas pelo custo de oportunidade e, a menos que haja uma nova informação, tarefas atuais deverão ser identificadas para que sejam interrompidas, de modo a adequar novos trabalhos. Isto pode necessitar de uma posição mais assertiva dos generalistas do que o que talvez estejamos acostumados. Precisamos nos impor e dar forma à agenda clínica a partir de nossa perspectiva individual, pautando o trabalho, as fontes da atenção primária na academia e o mundo da medicina com base em evidências. Nosso papel como implementadores passivos de ideias de saúde pública e de especialistas deve ser atualizado. Para fazê-lo, precisamos de um papel mais forte no processo criativo de síntese de evidência e criação de políticas. Precisamos garantir que a "voz" comum seja representada.

Deveríamos recrutar os criadores de protocolos para garantir que os generalistas (MFC) com uma perspectiva tão valiosa e ampla, sejam habilitados a ter uma influência maior em sua produção.

Os generalistas que estão na linha de frente (MFC) são clínicos gerais valiosos, e invocamos aqueles que possam pensar que suas vozes não são importantes para se envolverem no ato de moldar o futuro de nossa prática clínica para nossos pacientes e para nós mesmos.

\section{Referências}

1. Moynihan R, Glasziou P, Woloshin S, et al. Winding back the harms of too much medicine. ("Minimizando os danos causados por excesso de medicamentos"). BMJ 2013; 346: f1271. DOI: http://dx.doi.org/10.1136/bmj.f1271

2. Carter SM, RogersW, Heath I, et al. The challenge of overdiagnosis begins with it definition. ("O desafio do sobrediagnóstico começa com sua definição"). BMJ 2015; 350: h869. DOI: http://dx.doi.org/10.1136/bmj.h869

3. Heath I. Overdiagnosis: when good intentions meet vested interests - an essay. ("Sobrediagnóstico: quando boas intenções encontram interesses ocultos"). BMJ 2013; 347: f6361. DOI: http://dx.doi.org/10.1136/bmj.f6361

4. National Institute for Health and Care Excellence. Costing report: lipid modification. Implementing the NICE guideline on lipid modification (CG181). ("Instituto Nacional para Excelência em Saúde e Cuidado: Relatório de custeio: modificação de lipídio. Implementando as diretrizes do NICE sobre a modificação de lipídios"). July 2014. https://www.nice.org.uk/ guidance/cg181/resources/lipid-modification-update-costing-report 243777565 (accessed 1 Feb 2016).

5. Krogsbøll T, Jørgensen K, Grønhøj Larsen C, Gøtzsche PC. General health checks in adults for reducing morbidity and mortality from disease. ("Exames de saúde gerais em adultos para reduzir a moribilidade e mortalidade causadas por doenças"). Cochrane Database Syst Rev 2012;10: CD009009.

6. Jørgensen T, Jacobsen RK, Toft $U$, et al. Effect of screening and lifestyle counselling on incidence of ischaemic heart disease in general population: Inter99 randomised trial. ("O efeito de triagens e aconselhamento de estilo de vida sobre a incidência de doença cardíaca isquémica na população geral: ensaio randomizado Inter99") BMJ 2014; 348: g3617. 
7. Capewell S, McCartney M, Holland W. Invited debate: NHS Health Checks - a naked emperor? ("Debate: Exames de Saúde do SNS - um emperador nu?") J Public Health 2015; 37(2): 187-192. DOI: http://dx.doi.org/10.1093/pubmed/fdv063

8. The UK National Screening Committee. The UK NSC recommendation on Screening for Dementia. ("O Comitê de Triagem Nacional do Reino Unido. A recomendação do CTN do RU sobre a Triagem para Demência").2015. http://legacy.screening. nhs.uk/dementia (accessed 5 Feb 2016).

9. Yudkin JS, Montori VM. The epidemic of pre-diabetes: the medicine and the politics. ("A epidemia de pré-diabetes: a medicina e a política"). BMJ2014; 349: g4485.

10. Moynihan R, Glassock R, Doust J. Chronic kidney disease controversy: how expanding definitions are unnecessarily labelling many people as diseased. ("A controvérsia da doença crônica renal: como a expansão de definições está rotulando desnecessariamente muitas pessoas como doentes"). BMJ 2013; 347: f4298. DOI: http://dx.doi.org/10.1136/bmj.f4298

11. National Institute for Health and Care Excellence. Wider use of statins could cut deaths from heart disease. 2014. ("Instituto Nacional para Excelência em Saúde e Cuidado. Uso mais amplo de estatinas pode reduzir mortes por doenças cardíacas"). (https://www.nice.org.uk/news/article/wider-use-of-statins-could-cut-deaths-from-heart-disease (accessed 5 Feb 2016).

12. Royal College of General Practitioners. RCGP Standing Group on Overdiagnosis. For shared decisions in healthcare. ("Grupo Permanente sobre Sobrediagnóstico de RCGP. Por decisões compartilhadas no atendimento médico"). http:// www.rcgp.org.uk/policy/rcgp-policy-areas/ /media/Files/Policy/A-Z-policy/2015/C72 Standing Groupon Over-diagnosis revise 2.ashx (accessed 5 Feb 2016). 